\title{
ON THE BASS STABLE RANK OF STEIN ALGEBRAS
}

\author{
ALEXANDER BRUDNYI
}

\begin{abstract}
We compute the Bass stable rank of the ring $\Gamma\left(X, \mathcal{O}_{X}\right)$ of global sections of the structure sheaf $\mathcal{O}_{X}$ on a finite-dimensional Stein space $\left(X, \mathcal{O}_{X}\right)$ and then apply this result to the problem of the factorization of invertible holomorphic matrices on $X$.
\end{abstract}

\section{Formulation of Main Results}

1.1. Let $A$ be an associative ring with identity 1 . An element $a=\left(a_{1}, \ldots, a_{n}\right) \in A^{n}$ is unimodular if there exists $b=\left(b_{1}, \ldots, b_{n}\right) \in A^{n}$ such that $\langle b, a\rangle=\sum_{i=1}^{n} b_{i} a_{i}=1$. By $U_{n}(A) \subset A^{n}$ we denote the set of unimodular elements of $A^{n}$. An element $a=\left(a_{1}, \ldots, a_{n}\right) \in$ $U_{n}(A)$ is said to be reducible if there exist $c_{1}, \ldots, c_{n-1} \in A$ such that

$$
\left(a_{1}+c_{1} a_{n}, \ldots, a_{n-1}+c_{n-1} a_{n}\right) \in U_{n-1}(A) .
$$

$A$ is said to have a stable rank at most $n-1$ if every $a \in U_{n}(A)$ is reducible. The stable rank of $A$, denoted by $\operatorname{sr}(A)$, is the least $n-1$ with this property.

The concept of the stable rank introduced by Bass [B] plays an important role in some stabilization problems of algebraic $K$-theory analogous to that of dimension in topology. Despite a simple definition, $\operatorname{sr}(A)$ is often quite difficult to calculate even for relatively uncomplicated rings $A$ (cf. [V1]). In this note we compute the stable rank of the ring $\Gamma\left(X, \mathcal{O}_{X}\right)$ of global sections of the structure sheaf $\mathcal{O}_{X}$ on a finite-dimensional Stein space $\left(X, \mathcal{O}_{X}\right)$. (For basic facts about Stein spaces we refer the readers to the book GR.) Our research is motivated by the recent work of Ivarsson and Kutzschebauch [IK1], IK2] which solves the Vaserstein problem on the factorization of invertible holomorphic matrices on a finite-dimensional reduced Stein space posed by Gromov $G$, see section 1.2 below.

Recall that for a finite-dimensional complex analytic space $\left(X, \mathcal{O}_{X}\right)$ there is a natural algebra homomorphism ^ ${ }^{\wedge} \Gamma\left(X, \mathcal{O}_{X}\right) \rightarrow C(X)$ with image $\mathcal{O}(X)$ the ring of holomorphic functions on $X$, injective if $\left(X, \mathcal{O}_{X}\right)$ is reduced. A space $\left(X, \mathcal{O}_{X}\right)$ is said to be Stein if it is holomorphically convex (i.e., for each infinite discrete set $D \subset X$ there exists $f \in \mathcal{O}(X)$ which is unbounded on $D$ ) and holomorphic separable (i.e., for all $x, y \in X, x \neq y$, there exists $f \in \mathcal{O}(X)$ such that $f(x) \neq f(y))$. In what follows, sometimes for brevity we omit the structure sheaf $\mathcal{O}_{X}$ in the notation of the Stein space, i.e., write $X$ instead of $\left(X, \mathcal{O}_{X}\right)$. By $\operatorname{dim} X$ we denote the complex dimension of $X$.

2010 Mathematics Subject Classification. Primary 32E10. Secondary 18F25.

Key words and phrases. Stable rank, Stein space, holomorphic function, Cartan's theorems, Jacobson radical, invertible matrix.

Research supported in part by NSERC. 
Our main result reads as follows.

Theorem 1.1. Let $\left(X, \mathcal{O}_{X}\right)$ be a finite-dimensional Stein space. Then

$$
\operatorname{sr}\left(\Gamma\left(X, \mathcal{O}_{X}\right)\right)=\operatorname{sr}(\mathcal{O}(X))=\left\lfloor\frac{1}{2} \operatorname{dim} X\right\rfloor+1 .
$$

The particular case of the theorem for $X=\mathbb{C}$ was proved earlier in [Ru and [CS1].

1.2. In this part using Theorem 1.1 we extend and sharpen some results of [IK1], [IK2].

For an associative ring $A$ with 1 by $M_{k, n}(A)$ we denote the set of $k \times n$ matrices with entries in $A$, by $G L_{n}(A) \subset M_{n, n}(A)$ the group of invertible matrices, and by $S L_{n}(A) \subset$ $G L_{n}(A)$ the subgroup of matrices with determinant 1.

Recall that a matrix in $S L_{n}(A)$ is said to be elementary if it differs from the identity matrix $I_{n}$ by at most one non-diagonal entry.

By $E_{n}(A)$ we denote the subgroup of $S L_{n}(A)$ generated by all elementary matrices.

Let $t_{n}(A)$ denote the minimal number $t$ such that every matrix in $E_{n}(A)$ is the product of $t$ matrices such that each of them is either upper triangular with 1 along the main diagonal or lower triangular with 1 along the diagonal.

It is well-known that if $A$ is a field, then $E_{n}(A)=S L_{n}(A)$ and each $t_{n}(A)<\infty$. In general this is not always true. For instance, for $A=\mathbb{F}\left[x_{1}, \ldots, x_{d}\right]$, the ring of polynomials in $d$ indeterminates over a field, $E_{2}(A) \subsetneq S L_{2}(A)$ if $d \geq 2$, see [C, Prop. (7.3)], but $E_{n}(A)=S L_{n}(A)$ for all $d$ and $n \geq 3$, see [S, Cor.6.7]1] However, even in this case $t_{n}(A)=\infty$ if $\mathbb{F}$ is of infinite transcendence degree over its prime field, see [W], Prop. (1.5)].

Further, it was proved by Vaserstein in [V3, Th.4] that for $A=C(X)$, the algebra of complex-valued continuous functions on a $d$-dimensional normal topological space $X$, $E_{n}(A)$ coincides with the set of null-homotopic maps, i.e., maps in $S L_{n}(A):=C\left(X, S L_{n}(\mathbb{C})\right)$ homotopic (in this class of maps) to a constant map, and, moreover, there exists a constant $v(d) \in \mathbb{N}$ depending on $d$ only such that $\sup _{n} t_{n}(A) \leq v(d)$ (see also [DV, Lm. 7]).

A similar problem for the algebra $\mathcal{O}(X)$, where $X$ is a finite-dimensional reduced Stein space, was posed by Gromov in [G, 3.5.G] (the paper is devoted to the extension of the classical Oka-Grauert theorem) and solved recently by Ivarsson and Kutzschebauch in [IK1. (see also [IK2]) based on [V3, Th.4] and [F, Th. 8.3].

Specifically, they proved that $E_{n}(\mathcal{O}(X))$ coincides with the set of null-homotopic holomorphic maps of $S L_{n}(\mathcal{O}(X)):=\mathcal{O}\left(X, S L_{n}(\mathbb{C})\right)$ and that the number $t_{n}(\mathcal{O}(X))$ is bounded from above by a constant depending on $n$ and $d:=\operatorname{dim} X$ tending to $\infty$ as $n \rightarrow \infty$.

Remark 1.2. Note that [IK2, Th. 3.1] implies that $t_{2}(\mathcal{O}(X)) \leq v(2 d)+2$ with $v(\cdot)$ as in [V3, Th. 4] above. This and [DV, Lm. 7] produce a much better upper bound $\sup _{n} t_{n}(\mathcal{O}(X)) \leq$ $v(2 d)+3$.

In addition, [DV, Lm.7], Theorems 5.1, 5.2 and Proposition 4.1 of [IK2 imply that $t_{n}(\mathcal{O}(X))=4$ for all $n \geq 2$ if $d=1$ and $\sup _{n} t_{n}(\mathcal{O}(X))=5$ if $d=2$. (In [IK2] similar statements are formulated for $t_{2}(\mathcal{O}(X))$ only.)

\footnotetext{
${ }^{1}$ Note that for $d=1, E_{n}(A)=S L_{n}(A)$ for all $n$ as $A$ is an Euclidean ring.
} 
Now, let us consider the case of the algebra $\Gamma\left(X, \mathcal{O}_{X}\right)$ for a finite-dimensional Stein space $X$. Clearly, the algebra homomorphism ^ $: \Gamma\left(X, \mathcal{O}_{X}\right) \rightarrow \mathcal{O}(X)$ induces a group homomorphism ${ }_{n}: S L_{n}\left(\Gamma\left(X, \mathcal{O}_{X}\right)\right) \rightarrow S L_{n}(\mathcal{O}(X)),\left(f_{i j}\right) \mapsto\left(\hat{f}_{i j}\right)$.

Using the main result of [IK1], some results of [DV] and Theorem 1.1 we prove

\section{Proposition 1.3.}

(1) $H \in E_{n}\left(\Gamma\left(X, \mathcal{O}_{X}\right)\right)$ if and only if $\widehat{H}_{n} \in E_{n}(\mathcal{O}(X))$. Moreover,

$$
\sup _{n} t_{n}\left(\Gamma\left(X, \mathcal{O}_{X}\right)\right) \leq v(2 d)+5
$$

where $d:=\operatorname{dim} X$.

(2) There is a number $n(d) \in \mathbb{N}$ such that for all $n \geq n(d)$

$$
t_{n}\left(\Gamma\left(X, \mathcal{O}_{X}\right)\right) \leq 6 .
$$

(3) If $d \in\{1,2\}$, then $S L_{n}\left(\Gamma\left(X, \mathcal{O}_{X}\right)\right)=E_{n}\left(\Gamma\left(X, \mathcal{O}_{X}\right)\right)$ for all $n$ and

$$
t_{n}\left(\Gamma\left(X, \mathcal{O}_{X}\right)\right)=4 \text { for all } n \geq 2 \text { if } d=1 \text { and } \sup _{n} t_{n}\left(\Gamma\left(X, \mathcal{O}_{X}\right)\right) \leq 7 \quad \text { if } \quad d=2 \text {. }
$$

\section{Auxiliary Results}

In this section we collect some results used in the proof of Theorem 1.1.

2.1. Let $\left(X, \mathcal{O}_{X}\right)$ be a finite-dimensional Stein space. By the Cartan and Oka theorem, the nilradical $\mathfrak{n}\left(\mathcal{O}_{X}\right)$ of $\mathcal{O}_{X}$ (i.e., the union of nilradicals of stalks $\left.\mathcal{O}_{x}, x \in X\right)$ is a coherent sheaf of ideals on $X$ and so by Cartan's Theorem B we have the following exact sequence of global sections of sheaves

$$
0 \rightarrow \Gamma\left(X, \mathfrak{n}\left(\mathcal{O}_{X}\right)\right) \rightarrow \Gamma\left(X, \mathcal{O}_{X}\right) \stackrel{r^{*}}{\rightarrow} \Gamma\left(X, \mathcal{O}_{\text {red } X}\right) \rightarrow 0,
$$

where $\mathcal{O}_{\text {red } X}:=\mathcal{O}_{X} / \mathfrak{n}\left(\mathcal{O}_{X}\right)$ is the structure sheaf on the reduction of $X$.

Lemma 2.1. $\Gamma\left(X, \mathfrak{n}\left(\mathcal{O}_{X}\right)\right)$ is the Jacobson radical of $\Gamma\left(X, \mathcal{O}_{X}\right)$.

Proof. By definition, the Jacobson radical $\mathscr{J}(A)$ of a commutative ring $A$ is the intersection of all maximal ideals of $A$ and the Jacobson radical of $A / \mathscr{J}(A)$ is trivial. In our case, if $f \in \mathscr{J}\left(\Gamma\left(X, \mathcal{O}_{\operatorname{red} X}\right)\right)$, then $\hat{f}(x)=0$ for all $x \in X$, i.e., $\hat{f}=0 \in \mathcal{O}(X)$. Since ^ : $\Gamma\left(X, \mathcal{O}_{\operatorname{red} X}\right) \rightarrow \mathcal{O}(X)$ is an isomorphism of algebras, $f=0$. Thus $\mathscr{J}\left(\Gamma\left(X, \mathcal{O}_{\operatorname{red}} X\right)\right)=\{0\}$ and due to (2.1), $\mathscr{J}\left(\Gamma\left(X, \mathcal{O}_{X}\right)\right) \subset \Gamma\left(X, \mathfrak{n}\left(\mathcal{O}_{X}\right)\right)$.

Next, let $g \in \Gamma\left(X, \mathfrak{n}\left(\mathcal{O}_{X}\right)\right)$. In order to check that $g$ is in the Jacobson radical one must prove that $1-f g$ is invertible for all $f \in \Gamma\left(X, \mathcal{O}_{X}\right)$, see, e.g., [AM, Prop. 1.9]. Indeed, by the definition of the nilradical, there exist a locally finite open cover $\left(U_{i}\right)_{i \in I}$ of $X$ and a family $\left\{n_{i}\right\}_{i \in I} \subset \mathbb{N}$ such that $\left.g^{n_{i}}\right|_{U_{i}}=0, i \in I$. This implies that $h:=\sum_{i=0}^{\infty}(f g)^{i}$ is a well-defined section of $\Gamma\left(X, \mathcal{O}_{X}\right)$. Moreover, for each $i \in I$,

$$
\left.\left.(1-f g)\right|_{U_{i}} \cdot h\right|_{U_{i}}=\left.\left.(1-f g)\right|_{U_{i}} \cdot \sum_{k=0}^{n_{i}-1}(f g)^{k}\right|_{U_{i}}=\left.\left(1-(f g)^{n_{i}}\right)\right|_{U_{i}}=1 .
$$

This shows that $(1-f g) \cdot h=1$, as required. Thus $g \in \mathscr{J}\left(\Gamma\left(X, \mathcal{O}_{X}\right)\right)$ and so $\Gamma\left(X, \mathfrak{n}\left(\mathcal{O}_{X}\right)\right) \subset$ $\mathscr{J}\left(\Gamma\left(X, \mathcal{O}_{X}\right)\right)$.

This completes the proof of the lemma. 
Remark 2.2. (1) Arguing as in the proof of the lemma one obtains that if $s(t)=\sum_{i=0}^{\infty} c_{i} t^{i}$ is a formal power series in $t$, then $s(g)$ is a well-defined section of $\Gamma\left(X, \mathcal{O}_{X}\right)$ for each $g \in \Gamma\left(X, \mathfrak{n}\left(\mathcal{O}_{X}\right)\right)$.

(2) Due to Lemma 2.1, [V1, Lm. 3] and the fact that $\mathcal{O}(X)$ is isomorphic to $\Gamma\left(X, \mathcal{O}_{\operatorname{red} X}\right)$,

$$
\operatorname{sr}(\mathcal{O}(X))=\operatorname{sr}\left(\Gamma\left(X, \mathcal{O}_{\operatorname{red} X}\right)\right)=\operatorname{sr}\left(\Gamma\left(X, \mathcal{O}_{X}\right)\right) .
$$

Moreover, if $X_{i} \subset X, i \in \mathbb{N}$, are connected components of $X$, then each $\left(X_{i}, \mathcal{O}_{X_{i}}\right)$, $\mathcal{O}_{X_{i}}:=\left.\mathcal{O}_{X}\right|_{X_{i}}$, is Stein and $\Gamma\left(X, \mathcal{O}_{X}\right)$ is isomorphic to the direct product of the algebras $\Gamma\left(X_{i}, \mathcal{O}_{X_{i}}\right), i \in \mathbb{N}$. In particular, cf. [V1, Lm.2],

$$
\operatorname{sr}\left(\Gamma\left(X, \mathcal{O}_{X}\right)\right)=\sup _{i \in \mathbb{N}} \operatorname{sr}\left(\Gamma\left(X, \mathcal{O}_{X_{i}}\right)\right)
$$

In view of (2.2), (2.3), without loss of generality we may assume that $\left(X, \mathcal{O}_{X}\right)$ in the hypothesis of Theorem 1.1 is a connected reduced Stein space.

2.2. We retain the notation of the previous section. For a commutative unital algebra $A$ by $A^{-1} \subset A$ we denote the multiplicative group of invertible elements.

\section{Lemma 2.3.}

$$
\left(r^{*}\right)^{-1}\left(\Gamma\left(X, \mathcal{O}_{\operatorname{red} X}\right)^{-1}\right)=\Gamma\left(X, \mathcal{O}_{X}\right)^{-1} .
$$

Proof. Let $f, f^{\prime} \in \Gamma\left(X, \mathcal{O}_{X}\right)$ be such that $r^{*}(f):=g \in \Gamma\left(X, \mathcal{O}_{\text {red } X}\right)^{-1}$ and $r^{*}\left(f^{\prime}\right)=g^{-1}$, cf. (2.1). Since $r^{*}$ is an algebra homomorphism, $r^{*}\left(1-f f^{\prime}\right)=0$, i.e., $u:=1-f f^{\prime} \in$ $\Gamma\left(X, \mathfrak{n}\left(\mathcal{O}_{X}\right)\right)$. As in the proof of Lemma 2.1, $v:=\sum_{i=0}^{\infty} u^{i}$ is a well-defined section of $\Gamma\left(X, \mathfrak{n}\left(\mathcal{O}_{X}\right)\right)$ inverse to $1-u$. Hence, $f \cdot\left(f^{\prime} v\right)=1$, i.e., $f \in \Gamma\left(X, \mathcal{O}_{X}\right)^{-1}$.

Lemma 2.4. Suppose $A \in G L_{m}\left(\Gamma\left(X, \mathcal{O}_{X}\right)\right)$ is such that all entries of $I_{m}-A$ belong to $\Gamma\left(X, \mathfrak{n}\left(\mathcal{O}_{X}\right)\right)$. Then $A$ is a product of $(m+4)(m-1)$ elementary matrices and a matrix $\exp (h) \cdot I_{m}$ for some $h \in \Gamma\left(X, \mathfrak{n}\left(\mathcal{O}_{X}\right)\right)$.

Proof. Clearly, $\operatorname{det} A=1-g$ for some $g \in \Gamma\left(X, \mathfrak{n}\left(\mathcal{O}_{X}\right)\right)$. Thus $\log (1-g):=\sum_{i=1}^{\infty} \frac{g^{i}}{i} \in$ $\Gamma\left(X, \mathfrak{n}\left(\mathcal{O}_{X}\right)\right)$, cf. Remark 2.2, Similarly, $\exp (\log (1-g)):=\sum_{i=0}^{\infty} \frac{(\log (1-g))^{i}}{i !} \in \Gamma\left(X, \mathcal{O}_{X}\right)$ and it is readily seen that $\exp (\log (1-g))=1-g$. We set

$$
h:=\frac{\log (1-g)}{m} .
$$

Then $A=\exp (h) \cdot I_{m} \cdot \tilde{A}$, where $\tilde{A}$ satisfies the hypotheses of the lemma and $\operatorname{det} \tilde{A}=1$. Applying to $\tilde{A}$ the Gauss-Jordan elimination process using only addition operations we present $\tilde{A}$ as a product of $m(m-1)$ elementary matrices and a diagonal matrix $D$, $\operatorname{det} D=$ 1 , satisfying the hypotheses of the lemma. Due to the Whitehead lemma, $D$ is a product of $4(m-1)$ elementary matrices.

This proves the required statement. 
2.3. Let $\left(X, \mathcal{O}_{X}\right)$ be a reduced Stein space of complex dimension $n$ and $F \in M_{m, l}(\mathcal{O}(X))$ be a holomorphic $m \times l$ matrix with $1 \leq l<m$.

Lemma 2.5. Suppose the family of all minors of order $l$ of $F$ does not have common zeros. If $m-l \geq \frac{n}{2}$, then $F$ can be extended to a matrix in $G L_{m}(\mathcal{O}(X))$.

Proof. The matrix $F$ determines a trivial holomorphic subbundle $\xi$ of rank $l$ of the trivial holomorphic vector bundle $\theta^{m}:=X \times \mathbb{C}^{m}$ on $X$ (so that the columns of $F$ are holomorphic sections of $\xi$ linear independent at each point of $X$ ). Let $\theta^{m} / \xi$ be the holomorphic quotient bundle. Since $X$ is Stein, by Cartan's Theorem B there exists a holomorphic subbundle $\eta$ of $\theta^{m}$ isomorphic to $\theta^{m} / \xi$ such that $\xi \oplus \eta=\theta^{m}$. Thus, $\eta$ is stably trivial. Since, due to [H], $X$ is homotopic to a $C W$ complex of dimension $n$ and rank $\eta=m-l \geq \frac{n}{2}$, bundle $\eta$ is (topologically) trivial, see, e.g., [Hus, Th.3.4.7, 9.1.5]. Therefore, by the Grauert theorem, $\eta$ is holomorphically trivial. In global coordinates of $\theta^{m}$ holomorphic sections $s_{1}, \ldots, s_{m-l}$ trivializing $\eta$ determine an $(m-l) \times m$ matrix $F^{\prime}$ such that $\left(F, F^{\prime}\right) \in G L_{m}(\mathcal{O}(X))$ is an invertible holomorphic matrix extending $F$.

2.4. Let $A$ be a commutative ring with identity 1 and $J(a) \subset A$ be the principal ideal generated by $a \in A$.

Lemma 2.6. An element of the form $\left(a_{1}, \ldots, a_{n}, a\right) \in U_{n+1}(A), n \in \mathbb{N}$, is reducible if and only if the map $U_{n}(A) \rightarrow U_{n}(A / J(a))$ induced by the quotient homomorphism is surjective.

Proof. The proof is straightforward, see, e.g., the proof of the Proposition in [CL, Sect. 3].

\section{Proof of Theorem 1.1}

Proof. As it was mentioned in Remark 2.2(2), it suffices to prove the theorem for a connected finite-dimensional reduced Stein space $\left(X, \mathcal{O}_{X}\right)$. (In this case, $\Gamma\left(X, \mathcal{O}_{X}\right) \cong \mathcal{O}(X)$.) Under this assumption we set

$$
s(X):=\left\lfloor\frac{1}{2} \operatorname{dim} X\right\rfloor+1
$$

First, we prove that

$$
\operatorname{sr}(\mathcal{O}(X)) \leq s(X)
$$

To this end we need to check the following statement.

Claim. Each element $\left(f_{1}, \ldots, f_{s(X)}, f\right) \in U_{s(X)+1}\left(\mathcal{O}_{X}\right)$ is reducible.

This is obvious for $f=0$ or $f \in \mathcal{O}(X)^{-1}$. Next, assuming that $f \notin \mathcal{O}(X)^{-1} \cup\{0\}$ by $\mathcal{Z}(f) \subset X$ we denote its zero locus. Since $X$ is connected, there exists an irreducible component $\tilde{X}$ of $X$ such that the complex analytic subset $\mathcal{Z}(f) \cap \tilde{X}$ of $\tilde{X}$ has dimension $\operatorname{dim} \tilde{X}-1(\leq \operatorname{dim} X-1)$. By $X_{f}$ we denote the union of all such components $\tilde{X}$ and all irreducible components $\bar{X}$ of $X$ such that $\mathcal{Z}(f) \cap \bar{X}=\emptyset$. Let $X^{\prime}:=\operatorname{cl}\left(X \backslash X_{f}\right)$. Then $\left(X_{f}, \mathcal{O}_{X_{f}}\right), \mathcal{O}_{X_{f}}:=\left.\mathcal{O}_{X}\right|_{X_{f}}$, and $\left(X^{\prime}, \mathcal{O}_{X^{\prime}}\right), \mathcal{O}_{X^{\prime}}:=\left.\mathcal{O}_{X}\right|_{X^{\prime}}$, are complex analytic subspaces of $X$ and $\left.f\right|_{X^{\prime}}=0$. 
Lemma 3.1. If an element $\left(f_{1}, \ldots, f_{s(X)}, f\right) \in U_{s(X)+1}(\mathcal{O}(X))$ is reducible over $X_{f}$, i.e., there exist $h_{1}, \ldots, h_{s(X)} \in \mathcal{O}\left(X_{f}\right)$ such that

$$
\left(\left.f_{1}\right|_{X_{f}}-\left.h_{1} f\right|_{X_{f}}, \ldots,\left.f_{s(X)}\right|_{X_{f}}-\left.h_{s(X)} f\right|_{X_{f}}\right) \in U_{s(X)}\left(\mathcal{O}\left(X_{f}\right)\right),
$$

then it is reducible.

Proof. Due to Cartan's Theorem B for $X$ there are some $h_{i}^{\prime} \in \mathcal{O}(X)$ such that $\left.h_{i}^{\prime}\right|_{X_{f}}=h_{i}$, $i=1, \ldots, s(X)$. From here, (3.3) and the fact that $\left.f\right|_{X^{\prime}}=0$ we obtain that the family of holomorphic functions $\left\{f_{i}-h_{i}^{\prime} f\right\}_{1 \leq i \leq s(X)}$ does not have common zeros. Therefore by the corona theorem for Stein spaces

$$
\left(f_{1}-h_{1}^{\prime} f, \ldots, f_{s(X)}-h_{s(X)}^{\prime} f\right) \in U_{s(X)}(\mathcal{O}(X)) .
$$

Thus $\left(f_{1}, \ldots, f_{s(X)}, f\right) \in U_{s(X)+1}(\mathcal{O}(X))$ is reducible.

Let $J(f) \subset \mathcal{O}\left(X_{f}\right)$ be the principal ideal generated by $\left.f\right|_{X_{f}}$. In order to confirm the Claim, due to Lemmas 2.6 and 3.1, it suffices to prove the following result.

Proposition 3.2. The map $U_{s(X)}\left(\mathcal{O}\left(X_{f}\right)\right) \rightarrow U_{s(X)}\left(\mathcal{O}\left(X_{f}\right) / J(f)\right)$ induced by the quotient homomorphism is surjective.

Proof. Let $\mathcal{Z}^{\prime}(f):=\mathcal{Z}(f) \cap X_{f}$ and $\mathcal{J}_{f} \subset \mathcal{O}_{X_{f}}$ be the sheaf of principal ideals generated by germs of $f$. Consider the complex analytic space $\left(\mathcal{Z}^{\prime}(f),\left.\left(\mathcal{O}_{X_{f}} / \mathcal{J}_{f}\right)\right|_{\mathcal{Z}^{\prime}(f)}\right)$. Its reduction is the complex analytic subspace $\left(\mathcal{Z}^{\prime}(f), \mathcal{O}_{\mathcal{Z}^{\prime}(f)}\right), \mathcal{O}_{\mathcal{Z}^{\prime}(f)}:=\left.\mathcal{O}_{X_{f}}\right|_{\mathcal{Z}^{\prime}(f)}$, of $X_{f}$. By $r$ we denote the corresponding reduction homomorphism of the structure sheaves. Applying Cartan's Theorem B to the long exact cohomology sequence obtained from the short sequence of sheaves

$$
0 \rightarrow \mathcal{J}_{f} \rightarrow \mathcal{O}_{X_{f}} \rightarrow \mathcal{O}_{X_{f}} / \mathcal{J}_{f} \rightarrow 0
$$

we get the following sequence

$$
0 \rightarrow \Gamma\left(X_{f}, \mathcal{J}_{f}\right) \rightarrow \Gamma\left(X_{f}, \mathcal{O}_{X_{f}}\right) \rightarrow \Gamma\left(X_{f}, \mathcal{O}_{X_{f}} / \mathcal{J}_{f}\right) \rightarrow 0
$$

Since $\mathcal{Z}^{\prime}(f)$ is of the complex codimension one in each irreducible component of $X_{f}$ having a nonvoid intersection with $\mathcal{Z}^{\prime}(f)$, under the natural identification of $\Gamma\left(X_{f}, \mathcal{O}_{X_{f}}\right)$ with $\mathcal{O}\left(X_{f}\right)$ the space $\Gamma\left(X_{f}, \mathcal{J}_{f}\right)$ coincides with $J(f)$. Similarly, since each section in $\Gamma\left(X_{f}, \mathcal{O}_{X_{f}} / \mathcal{J}_{f}\right)$ equals zero outside $\mathcal{Z}^{\prime}(f)$, the latter space is naturally identified with $\Gamma\left(\mathcal{Z}^{\prime}(f), \mathcal{O}_{X_{f}} / \mathcal{J}_{f}\right)$. These and (3.4) give the following exact sequence

$$
0 \rightarrow J(f) \rightarrow \mathcal{O}\left(X_{f}\right) \rightarrow \Gamma\left(\mathcal{Z}^{\prime}(f), \mathcal{O}_{X_{f}} / \mathcal{J}_{f}\right) \rightarrow 0 .
$$

Thus the statement of the proposition is equivalent to the following one:

$(*)$ The map $\phi: U_{s(X)}\left(\mathcal{O}\left(X_{f}\right)\right) \rightarrow U_{s(X)}\left(\Gamma\left(\mathcal{Z}^{\prime}(f), \mathcal{O}_{X_{f}} / \mathcal{J}_{f}\right)\right)$ induced by the quotient homomorphism and the restriction to $\mathcal{Z}^{\prime}(f)$ is surjective.

In turn, the reduction $r$ induces a homomorphism

$$
\left.r^{*}: \Gamma\left(\mathcal{Z}^{\prime}(f), \mathcal{O}_{X_{f}} / \mathcal{J}_{f}\right)\right) \rightarrow \mathcal{O}\left(\mathcal{Z}^{\prime}(f)\right),
$$

surjective due to Cartan's Theorem B. 
In the sequel, for a finite-dimensional complex vector space $V$ by $\mathfrak{r}^{*}$ we denote the linear $\left.\operatorname{map~id}_{V} \otimes r^{*}: V \otimes_{\mathbb{C}} \Gamma\left(\mathcal{Z}^{\prime}(f), \mathcal{O}_{X_{f}} / \mathcal{J}_{f}\right)\right) \rightarrow V \otimes_{\mathbb{C}} \mathcal{O}\left(\mathcal{Z}^{\prime}(f)\right)$. (The choice of $V$ will be understood from the context.) In particular, $\mathfrak{r}^{*}=\operatorname{id}_{\mathbb{C}^{s}(X)} \otimes r^{*}$ sends $U_{s(X)}\left(\Gamma\left(\mathcal{Z}^{\prime}(f), \mathcal{O}_{X_{f}} / \mathcal{J}_{f}\right)\right)$ to $U_{s(X)}\left(\mathcal{O}\left(\mathcal{Z}^{\prime}(f)\right)\right)$. Thus we obtain the following diagram

$$
U_{s(X)}\left(\mathcal{O}\left(X_{f}\right)\right) \stackrel{\phi}{\longrightarrow} U_{s(X)}\left(\Gamma\left(\mathcal{Z}^{\prime}(f), \mathcal{O}_{X_{f}} / \mathcal{J}_{f}\right)\right) \stackrel{\mathfrak{r}^{*}}{\longrightarrow} U_{s(X)}\left(\mathcal{O}\left(\mathcal{Z}^{\prime}(f)\right)\right)
$$

where $\mathfrak{r}^{*} \circ \phi$ is the map induced by the restriction to $\mathcal{Z}^{\prime}(f)$.

To check $(*)$, first, we prove

Lemma 3.3. The map $\mathfrak{r}^{*} \circ \phi: U_{s(X)}\left(\mathcal{O}\left(X_{f}\right)\right) \rightarrow U_{s(X)}\left(\mathcal{O}\left(\mathcal{Z}^{\prime}(f)\right)\right)$ is surjective.

Proof. Due to the corona theorem on a Stein space, sets $U_{s(X)}\left(\mathcal{O}\left(X_{f}\right)\right)$ and $U_{s(X)}\left(\mathcal{O}\left(\mathcal{Z}^{\prime}(f)\right)\right)$ coincide with $\mathcal{O}\left(X_{f},\left(\mathbb{C}^{s(X)}\right)^{*}\right)$ and $\mathcal{O}\left(\mathcal{Z}^{\prime}(f),\left(\mathbb{C}^{s(X)}\right)^{*}\right)$, respectively; here $\left(\mathbb{C}^{k}\right)^{*}:=\mathbb{C}^{k} \backslash\{0\}$. In turn, $\left(\mathbb{C}^{s(X)}\right)^{*}$ is homotopic to $\mathbb{S}^{2 s(X)-1}$ (the $(2 s(X)-1)$ - dimensional unit Euclidean sphere) while, due to $[\mathrm{H}], \mathcal{Z}^{\prime}(f)$ is homotopic to a $C W$ complex of dimension $\operatorname{dim} \mathcal{Z}_{f}^{\prime}(=$ $\left.\operatorname{dim} X_{f}-1 \leq \operatorname{dim} X-1<2 s(X)-1\right)$, see (3.1). Let us show that

Each map in $C\left(\mathcal{Z}^{\prime}(f),\left(\mathbb{C}^{s(X)}\right)^{*}\right)$ is extendable to a map in $C\left(X_{f},\left(\mathbb{C}^{s(X)}\right)^{*}\right)$.

Indeed, since the space $\left(\mathbb{C}^{s(X)}\right)^{*}$ is a simple absolute neighbourhood retract, in order to prove the previous statement it suffices to check that $H^{l+1}\left(X, \mathcal{Z}^{\prime}(f) ; \pi_{l}\right)=0$ for each $0<l<\operatorname{dim}\left(X_{f} \backslash \mathcal{Z}^{\prime}(f)\right)$, where $\pi_{l}$ is the $l$ th homotopy group of $\mathbb{S}^{2 s(X)-1}$, see, e.g., $[\mathrm{Hu}$, p. 348, (5.3)]. Clearly, this is true for $0<l \leq 2 s(X)-2$ (for $s(X)>1)$ because $\pi_{l}=0$ in this case. Next, if $l=2 s(X)-1+m$ for some $m \geq 0$, then from the long exact cohomological sequence of the pair $\left(X_{f}, \mathcal{Z}^{\prime}(f)\right)$ with coefficients in $\pi_{2 s(X)-1+m}$ we obtain

$$
\begin{aligned}
\cdots \rightarrow H^{2 s(X)-1+m}\left(\mathcal{Z}^{\prime}(f) ; \pi_{2 s(X)-1+m}\right) & \rightarrow H^{2 s(X)+m}\left(X_{f}, \mathcal{Z}^{\prime}(f) ; \pi_{2 s(X)-1+m}\right) \\
& \rightarrow H^{2 s(X)+m}\left(X_{f} ; \pi_{2 s(X)-1+m}\right) \rightarrow \cdots .
\end{aligned}
$$

The first term here is zero because $\mathcal{Z}^{\prime}(f)$ is homotopic to a $C W$ complex of dimension $<2 s(X)-1$ while the last term is zero because, due to $[\mathrm{H}], X_{f}$ is homotopic to a $C W$ complex of dimension $\operatorname{dim} X_{f} \leq \operatorname{dim} X \leq 2 s(X)-1$. Therefore the intermediate term of the above sequence is zero as well. This proves the required statement.

In particular, each map in $\mathcal{O}\left(\mathcal{Z}^{\prime}(f),\left(\mathbb{C}^{s(X)}\right)^{*}\right)$ extends to a map in $C\left(X_{f},\left(\mathbb{C}^{s(X)}\right)^{*}\right)$. Hence, by the Ramspott theorem $[\mathrm{R}]$ it extends also to a map in $\mathcal{O}\left(X_{f},\left(\mathbb{C}^{s(X)}\right)^{*}\right)$. This shows that $\mathfrak{r}^{*} \circ \phi$ is surjective and completes the proof of the lemma.

Now using the lemma let us prove the proposition.

Let $\mathcal{R}:=\Gamma\left(\mathcal{Z}^{\prime}(f), \mathfrak{n}\left(\mathcal{O}_{X_{f}} / \mathcal{J}_{f}\right)\right)$ denote the Jacobson radical of $\Gamma\left(\mathcal{Z}^{\prime}(f), \mathcal{O}_{X_{f}} / \mathcal{J}_{f}\right)$, see Lemma 2.1, Due to Lemma 3.3, given $h=\left(h_{1}, \ldots, h_{s(X)}\right) \in U_{s(X)}\left(\Gamma\left(\mathcal{Z}^{\prime}(f), \mathcal{O}_{X_{f}} / \mathcal{J}_{f}\right)\right.$ there exists $f=\left(f_{1}, \ldots, f_{s(X)}\right) \in U_{s(X)}\left(\mathcal{O}\left(X_{f}\right)\right)$ such that

$$
\mathfrak{r}^{*}(h)=\left.f\right|_{\mathcal{Z}^{\prime}(f)}\left(:=\mathfrak{r}^{*}(\phi(f))\right) .
$$

In order to prove $(*)$ we must show that there is some $\tilde{h} \in U_{s(X)}\left(\mathcal{O}\left(X_{f}\right)\right)$ such that $\phi(\tilde{h})=h$. 
Observe that by Lemma 2.5, $\mathfrak{r}^{*}(h)$ considered as a column-matrix can be extended to a matrix $\left(\mathfrak{r}^{*}(h), F\right) \in G L_{s(X)}\left(\mathcal{O}\left(\mathcal{Z}^{\prime}(f)\right)\right)$. Since, due to Cartan's Theorem B, the homomorphism $r^{*}: \Gamma\left(\mathcal{Z}^{\prime}(f), \mathcal{O}_{X_{f}} / \mathcal{J}_{f}\right) \rightarrow \Gamma\left(\mathcal{Z}^{\prime}(f), \mathcal{O}_{\mathcal{Z}^{\prime}(f)}\right) \cong \mathcal{O}\left(\mathcal{Z}^{\prime}(f)\right)$ induced by the reduction map is surjective, there is a matrix $\tilde{F} \in M_{s(X) \times(s(X)-1)}\left(\Gamma\left(\mathcal{Z}^{\prime}(f), \mathcal{O}_{X_{f}} / \mathcal{J}_{f}\right)\right)$ such that $\mathfrak{r}^{*}(\tilde{F})=F\left(\right.$ here $\mathfrak{r}^{*}=\operatorname{id}_{V} \otimes r^{*}$ with $\left.V:=M_{s(X) \times(s(X)-1)}(\mathbb{C})\right)$. Note that

$$
r^{*}(\operatorname{det}(h, \tilde{F}))=\operatorname{det}\left(\mathfrak{r}^{*}(h), F\right) \in \mathcal{O}\left(\mathcal{Z}^{\prime}(f)\right)^{-1} .
$$

Therefore by Lemma 2.3, $(h, F) \in G L_{s(X)}\left(\Gamma\left(\mathcal{Z}^{\prime}(f), \mathcal{O}_{X_{f}} / \mathcal{J}_{f}\right)\right)$.

Next, considering $\phi(f)$ as a column-matrix, we extend it to the $s(X) \times s(X)$ matrix $(\phi(f), \tilde{F})$. Since $\mathfrak{r}^{*}(h)=\mathfrak{r}^{*}(\phi(f))$, the matrix $(\phi(f), \tilde{F}) \in G L_{s(X)}\left(\Gamma\left(\mathcal{Z}^{\prime}(f), \mathcal{O}_{X_{f}} / \mathcal{J}_{f}\right)\right)$ and

$$
(h, \tilde{F}) \cdot(\phi(f), \tilde{F})^{-1}=: G
$$

is an invertible matrix of the form $I_{s(X)}+L$, where entries of $L$ belong to $\mathcal{R}$. Due to Lemma 2.4. $G$ is a product of a matrix $\exp (u) \cdot I_{s(X)}$ for some $u \in \mathcal{R}$ and finitely many elementary matrices with entries in $\Gamma\left(\mathcal{Z}^{\prime}(f), \mathcal{O}_{X_{f}} / \mathcal{J}_{f}\right)$. Due to (3.5), each matrix in the factorization of $G$ is the image of an invertible matrix, a multiple of $I_{s(X)}$ or an elementary matrix, with entries in $\mathcal{O}\left(X_{f}\right)$. Hence, there exists some $\tilde{G} \in G L_{s(X)}\left(\mathcal{O}\left(X_{f}\right)\right)$ such that $\Phi(\tilde{G})=G$, where $\Phi$ is defined by the application of $\phi$ to matrix columns. Then we have $(h, \tilde{F})=\Phi(\tilde{G}) \cdot(\phi(f), \tilde{F})$. In turn, this gives the identity of the first columns

$$
h=\Phi(\tilde{G}) \cdot \phi(f)=\phi(\tilde{G} \cdot f) .
$$

Since $\tilde{h}:=\tilde{G} \cdot f \in U_{s(X)}\left(\mathcal{O}\left(X_{f}\right)\right)$, the latter proves the surjectivity of $\phi$, cf. $\left(^{*}\right)$.

The proof of the proposition is complete.

As it was mentioned above, Lemmas 2.6 and 3.1 and Proposition 3.2 prove the Claim, i.e., $\operatorname{sr}(\mathcal{O}(X)) \leq s(X)$.

Now, to complete the proof of Theorem 1.1 let us show that

$$
s(X) \leq \operatorname{sr}(\mathcal{O}(X)) .
$$

In order to prove the inequality it suffices to point out an irreducible element of $U_{s(X)}(\mathcal{O}(X))$.

Let $\tilde{X} \subset X$ be an irreducible component of the maximal dimension $d(:=\operatorname{dim} X)$ and let $x \in \tilde{X}$ be a regular point. Due to Cartan's Theorems there exist holomorphic functions $f_{1}, \ldots, f_{d}$ on $X$ and an open neighbourhood $U$ of $x$ such that the holomorphic map $F=$ $\left(f_{1}, \ldots, f_{d}\right): X \rightarrow \mathbb{C}^{d}$ is one-to-one on $\operatorname{cl}(U)$, maps $x$ to 0 and $U$ biholomorphically onto the open unit ball $\mathbb{B}^{d} \subset \mathbb{C}^{d}$. Let $A(U)$ be the algebra of holomorphic functions on $U$ which extend continuously to its boundary. By our construction, $A(U)$ is isomorphic by means of the pullback of $\left.F\right|_{\mathrm{cl}(U)}$ to the (similarly defined) algebra $A\left(\mathbb{B}^{d}\right)$. Consider the element

$$
u=\left(z_{1}, z_{3}, \ldots, z_{2 s(X)-3}, p(z)\right) \in U_{s(X)}\left(\mathcal{O}\left(\mathbb{C}^{d}\right)\right), \quad z=\left(z_{1}, \ldots, z_{d}\right) \in \mathbb{C}^{d},
$$

where $p(z)=z_{1} z_{2}+z_{3} z_{4}+\cdots+z_{2 s(X)-3} z_{2 s(X)-2}-1$.

It was shown in the proof of [CS2, Th. 3.12] that $\left.u\right|_{\mathrm{cl}\left(\mathbb{B}^{d}\right)} \in U_{s(X)}\left(A\left(\mathbb{B}^{d}\right)\right)$ is irreducible. This implies that the restriction to $\operatorname{cl}(U)$ of $F^{*} u:=\left(f_{1}, f_{3}, \ldots, f_{2 s(X)-3}, p(F)\right) \in U_{s(X)}(\mathcal{O}(X))$ 
is irreducible (in $\left.U_{s(X)}(A(U))\right)$ and therefore $F^{*} u$ is irreducible. This proves inequality (3.7).

The proof of Theorem 1.1 is complete.

\section{Proof of Proposition 1.3}

(1) Let $H \in S L_{n}\left(\Gamma\left(X, \mathcal{O}_{X}\right)\right)$ be such that $\widehat{H}_{n} \in E_{n}(\mathcal{O}(X))$. Since, due to Cartan's Theorem B and Lemma 2.3, the homomorphism $\widehat{{ }_{n}}$ is surjective, the latter implies that $H=H^{1} \cdots H^{l} F$, where $H^{i}, 1 \leq i \leq l$, are alternating upper and lower triangular unipotent matrices in $S L_{n}\left(\Gamma\left(X, \mathcal{O}_{X}\right)\right)$ and $F=I_{n}+G$ for some matrix $G$ with entries in the Jacobson radical of $\left.\Gamma\left(X, \mathcal{O}_{X}\right)\right)$, see Lemma 2.1. By virtue of Lemma 2.4. $F \in E_{n}\left(\Gamma\left(X, \mathcal{O}_{X}\right)\right)$. Hence, $H \in E_{n}\left(\Gamma\left(X, \mathcal{O}_{X}\right)\right)$ as well.

The converse statement asserting that if $H \in E_{n}\left(\Gamma\left(X, \mathcal{O}_{X}\right)\right)$ then $\widehat{H}_{n} \in E_{n}(\mathcal{O}(X))$ is obvious.

Next, let $u t_{n}\left(\Gamma\left(X, \mathcal{O}_{X}\right)\right)$ be the minimal number $t$ such that every matrix in $E_{n}\left(\Gamma\left(X, \mathcal{O}_{X}\right)\right)$ is a product of $t$ matrices such that each of them is either upper triangular with 1 along the main diagonal or lower triangular with 1 along the diagonal and the first matrix is upper triangular. Then we have for all $n \geq 2$, see [DV, Lm. 7],

$$
t_{n}\left(\Gamma\left(X, \mathcal{O}_{X}\right)\right) \leq u t_{n}\left(\Gamma\left(X, \mathcal{O}_{X}\right)\right) \leq u t_{2}\left(\Gamma\left(X, \mathcal{O}_{X}\right)\right) \leq t_{2}\left(\Gamma\left(X, \mathcal{O}_{X}\right)\right)+1 .
$$

Also, due to [IK2, Th. 3.1],

$$
t_{2}(\mathcal{O}(X)) \leq t_{2}(C(X))+2 \leq v(2 d)+2 .
$$

Once again, Cartan's Theorem B and Lemma 2.3 imply that the homomorphism

$$
\widehat{2}_{2}: S L_{2}\left(\Gamma\left(X, \mathcal{O}_{X}\right)\right) \rightarrow S L_{2}(\mathcal{O}(X))
$$

is surjective and its kernel $K_{2}$ consists of matrices with determinant one of the form $F=$ $I_{2}+G$, where all entries of $G$ belong to the Jacobson radical of $\Gamma\left(X, \mathcal{O}_{X}\right)$. Applying the Gauss-Jordan elimination process and then the Whitehead lemma to such $F$ we write it as a product $F=F_{1} F_{2} F_{3} F_{4}$, where $F_{i} \in K_{2}, 1 \leq i \leq 4$, are alternating upper and lower triangular unipotent matrices. Now, the surjectivity of $\widehat{2}$ and the normality of $K_{2}$ imply, in view of (4.2), that each $H \in E_{2}\left(\Gamma\left(X, \mathcal{O}_{X}\right)\right)$ has a form

$$
H=H^{1} F H^{2} \cdots H^{l},
$$

where $H^{i}, 1 \leq i \leq l$, are alternating upper and lower triangular unipotent matrices in $S L_{2}\left(\Gamma\left(X, \mathcal{O}_{X}\right)\right), F \in K_{2}$ and $l \leq v(2 d)+2$. Writing $F=F_{1} F_{2} F_{3} F_{4}$ with $\left\{F_{i}\right\} \subset K_{2}$ as above such that the matrices $H_{1}$ and $F_{1}$ are both either upper or lower triangular we get that the matrices $F_{4}$ and $H^{2}$ are both either upper or lower triangular as well. Hence, we represent $H$ as a product $\left(H^{1} F_{1}\right) F_{2} F_{3}\left(F_{4} H_{2}\right) H_{3} \cdots H_{l}$ of at most $v(2 d)+4$ triangular unipotent matrices in $S L_{2}\left(\Gamma\left(X, \mathcal{O}_{X}\right)\right)$. Together with (4.1) this gives the required inequality

$$
\sup _{n} t_{n}\left(\Gamma\left(X, \mathcal{O}_{X}\right)\right) \leq v(2 d)+5
$$

(2) Due to [IK1, Th.2.3], [DV, Th.20(b)], our Theorem 1.1] and inequalities (4.1), (4.2) $t_{n}\left(\Gamma\left(X, \mathcal{O}_{X}\right)\right) \leq 6$ for all $n \geq n(d):=\left(\left\lfloor\frac{d}{2}\right\rfloor+2\right) \cdot\left(\left\lfloor\frac{v(2 d)}{2}\right\rfloor+3\right)$. 
(3) Since by the result of $[\mathrm{H}]$ the space $X$ is homotopic to a $C W$ complex of dimension $d$ and the homotopy groups $\pi_{i}\left(S L_{n}(\mathbb{C})\right), i=1,2$, are trivial, for $d=1,2$ each map in $C\left(X, S L_{n}(\mathbb{C})\right)$ is null-homotopic, see, e.g., [Hu, p. 351, (7.4)]. Thus in these cases $S L_{n}\left(\Gamma\left(X, \mathcal{O}_{X}\right)\right)=E_{n}\left(\Gamma\left(X, \mathcal{O}_{X}\right)\right)$ for all $n$ by the first statement of part (1) of the proposition.

Next, if $d=1$, then, by Theorem 1.1, $\operatorname{sr}\left(\Gamma\left(X, \mathcal{O}_{X}\right)\right)=1$ so that $t_{n}\left(\Gamma\left(X, \mathcal{O}_{X}\right)\right) \leq 4$ for all $n \geq 2$ by [DV, Lm.9]. One easily shows that 4 in the previous inequalities is optimal because any nontrivial diagonal matrix in $S L_{n}\left(\Gamma\left(X, \mathcal{O}_{X}\right)\right), n \geq 2$, cannot be written as a product of less than four triangular unipotent matrices.

If $d=2$, then using [IK2, Th. 5.2] and arguments similar to those of the proof of part (1) above we obtain that $\sup _{n} t_{n}\left(\Gamma\left(X, \mathcal{O}_{X}\right)\right) \leq u t_{2}\left(\Gamma\left(X, \mathcal{O}_{X}\right)\right) \leq u t_{2}(\mathcal{O}(X))+2 \leq 7$. We leave the details to the readers.

The proof of the proposition is complete.

Acknowledgment. I thank the anonymous referee for useful comments.

\section{REFERENCES}

[AM] M. F. Atiyah, I. G. Macdonald, Introduction to commutative algebra, Addison-Wesley Publishing Co., 1969.

[B] H. Bass, $K$-theory and stable algebra, Publ. Mat. I.H.E.S. 22 (1964), 5-60.

[C] P. M. Cohn, On the structure of the $G L_{2}$ of a ring, Inst. Hautes Études Sci. Publ. Math. 30 (1966), $5-53$.

[CL] G. Corach and A. R. Larotonda, Stable range in Banach algebras, J. Pure Appl. Algebra 32 (1984), 289-300.

[CS1] G. Corach and F. Suárez, Stable rank in holomorphic function algebras, Illinois J. Math. 29 (1985), 627-639.

[CS2] G. Corach and F. Suárez, Dense morphisms in commutative Banach algebras, Trans. Amer. Math. Soc. 304 (2) (1987), 537-547.

[DV] R. K. Dennis and L. N. Vaserstein, On a question of M. Newman on the number of commutators, J. Algebra 118 (1988), 150-161.

[F] F. Forstnerič, The Oka principle for sections of stratified fiber bundles, Pure Appl. Math. Q. 6 (2010), 843-874.

[G] M. Gromov, Oka's principle for holomorphic sections of elliptic bundles, J. Amer. Math. Soc. 2 (1989), 851-897.

[GR] H. Grauert and R. Remmert, Theory of Stein spaces, Springer, Berlin, 1979.

[H] H. A. Hamm, Zum Homotopietyp Steinscher Räume, J. reine angew. Math. 338 (1983), 121-135.

[Hu] S. T. Hu, Mappings of a normal space into an absolute neighbourhood retract, Trans. Amer. Math. Soc. 64 (1948), 336-358.

[Hus] D. Husemoller, Fibre bundles, Springer-Verlag, New York, 1994.

[IK1] B. Ivarsson and F. Kutzschebauch, Holomorphic factorization of mappings into $S L_{n}(\mathbb{C})$, Ann. Math. 175 (2012), 45-69.

[IK2] B. Ivarsson and F. Kutzschebauch, On the number of factors in the unipotent factorization of holomorphic mappings into $S L_{2}(\mathbb{C})$, Proc. Amer. Math. Soc. 140 (2012), no. 3, 823-838.

[R] K. J. Ramspott, Stetige und holomorphe Schnitte in Bündeln mit homogener Faser, Math. Z. 89 (1965), $234-246$.

[Ru] L. A. Rubel, Solution of problem 6117, Amer. Math. Monthly 85 (1978), 505-506.

[S] A. A. Suslin, On the structure of the special linear group over rings of polynomials, Izv. Akad. Nauk SSSR Ser. Mat. 41 (2) (1977), 235-252. 
[V1] L. N. Vaserstein, The stable range for rings and the dimension of topological spaces, Functional Anal. Appl., vol. 5 (1971), 102-110.

[V3] L. N. Vaserstein, Reduction of a matrix depending on parameters to a diagonal form by addition operations, Proc. Amer. Math. Soc. 103 (1988), 741-746.

[W] W. van der Kallen, $S L_{3}(\mathbb{C}[X])$ does not have bounded word length. In: Algebraic $K$-theory, Part I (Oberwolfach, 1980), Lecture Notes in Math. 966, Springer (1982), 357-361.

Department of Mathematics and Statistics

UNIVERSITY OF CALGARY

Calgary, Alberta

$\mathrm{T} 2 \mathrm{~N} 1 \mathrm{~N} 4$

E-mail address: abrudnyi@ucalgary.ca 